\title{
Influence of Spatial Ability in Navigation: Using look-ahead breadcrumbs on the Web
}

\section{Ishtiaq Ahmed and James Blustein*}

Faculty of Computer Science, Dalhousie University, Halifax, NS, Canada Fax: +1 902-492-1517 E-mail: ishtiaq@cs.dal.ca E-mail: jamie@cs.dal.ca ${ }^{*}$ Corresponding author

\begin{abstract}
Spatial implications of the commonly used 'navigation' metaphor have lead many researchers to investigate the relation between individual differences and navigation. This study presents an exploratory survey on the influence of spatial ability, the most incisive aspect of individual difference for navigation, when people try to accomplish their goal in the information space. There are still different opinions about the potential of visual mediators for people with low spatial ability that help in refraining from the state of being disoriented or lost in the huge information content. The findings motivated us to design a research framework focusing a special visual look-ahead breadcrumb tool. Breadcrumbs are a type of navigational aid that help users from being lost in large websites by providing information about their location in the site hierarchy. The framework is presented after the preliminary discussion. The results from the experiment report that spatial ability influenced navigation efficiency on the Web. The effect of spatial ability was marginally significant on the use of Back button during navigation. However, there was no interaction effect found between spatial ability and the visual look-ahead breadcrumbs on the navigation performance of the users.
\end{abstract}

Keywords: Hypertext navigation, spatial ability, information space, information visualization, breadcrumbs.

Reference to this paper should be made as follows: Ahmed, I. and Blustein, J. ( $\mathrm{xxxx}$ ) 'Influence of Spatial Ability in Navigation: Using look-ahead breadcrumbs on The Web', Int. J. Web Based Communities, Vol. x, No. x, pp.xxx-xxx.

Biographical notes: Mr. Ahmed is a Masters of Computer Science student. Prof. Blustein is an assistant professor of Computer Science at Dalhousie University, Canada. His primary research interest is in hypertext interfaces to improve access to, and use of, information.

Copyright (c) 200x Inderscience Enterprises Ltd. 


\section{Introduction}

Spatial ability is the ability to perceive spatial patterns or to maintain orientation with respect to objects in space (Ekstrom et al., 1976). It is the cognition of spatial properties of the world such as location, size, distance, direction, shape, movement, etc. In the context of navigation, it is the perceptual ability on the information space based on user's previously acquired knowledge, information processing capability and motor capability on spatial properties in real world. Spatial ability is often cited as a good predictor of individual's performance in human- computer interactions (Egan, 1988; Stanney \& Salvendy, 1995). The influence is more eminent in the act of browsing or navigation in an interaction space, if it has somewhat spatial layout inherent in it. (Benyon \& Murray, 1993; Dahlbäck et al., 1996).

Identifying the model in which people conceptualize the whole interaction environment and the extent of spatiality in this model is yet to be resolved clearly. It is still needed to forecast the browsing patterns of people with varying spatial abilities. The basic premise behind the metaphor is that finding information in a significantly large information space is psychologically similar to navigating in real world space and, hence, promoting the existence of a mental architecture that conceptualize the information space in a spatial manner, may increase the affectiveness, efficiency and enjoyment of navigation. It is believed that navigation performance of people with low spatial ability can be improved by providing them with visual tool that assist in grasping the layout of the space (Robertson, 1997; Vicente \& Williges, 1988). Benyon \& Murray (1993) and Höök and Dahlbäck (1997) conducted studies that clearly showed that people with high spatial ability can visualize the organization of information better than people with low ability. Stanney \& Salvendy (1995), found that visual tool for hierarchical representation of information is an effective means by which to accommodate low spatial individuals in information seeking tasks. But, another work by Chen (2000) revealed that it is not the spatial ability rather experience with the system that matters effectively in the performance of navigation in information space. Another interesting study (Allen, 1998) reveals that spatial organization of information helps the low ability people with their way finding but, in some cases, negatively affects the high spatial ability people. Dillon \& Vaughan (1997) were somewhat sceptic, in their landmark article on information shape, on the rationale of looking at the information systems as a metaphor of real world space. Instead, Dillon suggested the idea of Information Shape, which, in his view, more suitable to express the users' actual mental and physical tasks in information seeking (Dillon \& Vaughan, 1997; Dillon, 1995). However, the contraposition of results and divergence in opinion call for more efforts like ours on this topic.

\section{Navigation in Information Space}

Navigation is the process of moving from one location to another and knowing the relative position in order to reach the desired destination in a spatial environment. It includes understanding, partitioning an environment, exploring it and finding the right way to the end from starting point (Benyon, 2001). In order to explain information space, Benyon (2001) introduced the idea of activity space. Activity space is the physical spatial environment in which people perform a certain 
activity. Information space is the structured collection of information consisting of various information artifact and signs from where subjects seek information for their activity space. According to results from the study by Dieberger (1997), visualizing an information space is necessary for effective navigation. That is why people often impose metaphors to information space to give it a visual structure. Apart from the large body of information contained in it, an information space can be envisioned as a set of nodes, each of which is an information unit or sign (van Dyke Parunak, 1989). The topology of these nodes makes it resemble a space that can be traversed. The complete structure takes a shape like a city or town where the way-finder is not forced to take any single path to reach a destination. In this sense, hypermedia is the most notorious example of an information space. Besides, newspapers, television and even a single autonomous computer or paper document can be treated as an information space. The evolution of the World Wide Web over past several years has been astounding. Due to its huge information content and complexity in navigation, salience and structure make it the most complex information space for information users. Navigation in information space, thus, can be defined as the process where user moves through the virtual information network by following links from node to node and senses position in the network relative to the destination node. Navigation and way finding are used as similar terms in many works when it comes in the context of electronic information space (Dahlbäck \& Lönqvist, 2000). Based on the size, orientation and structure, the navigation mechanism varies from one information space to other. There are numerous definitions for navigation in information space and Dahlbäck \& Lönqvist (2000) concluded it as a task rather synonymous to information retrieval.

\section{Spatial Ability}

Analyzing and understanding spatial ability is a complex affair in psychology. Every task that presents figural stimuli does not require spatial ability to exploit by the individual. Nor does the absence of a figural stimulus mean that there is no spatial processing (Lohman \& Kyllonen, 1983). Spatial ability or thinking, in general, requires several psychological attributes such as ability to encode information, remember, transform and differentiate spatial objects (Lohman \& Kyllonen, 1983). Kritchvesky's posits on spatial ability are quite acceptable to the community (Caplan \& Romans, 1998). According to him, there are five broad categories of spatial functions, which are perception, memory, attention, mental operations and construction. The functions, all together, contain nine basic spatial skills. These are object localization, line orientation detection, spatial synthesis, short term spatial memory, long term spatial memory, attention to left hemispace, attention to right hemispace, mental rotation and spatial construction. Other spatial properties proposed by various authors include visual scanning, face recognition, topographical orientation, and identification of incomplete figures and detection of hidden figures. Much research has been conducted to devise appropriate psychological tests to measure these abilities. Ekstrom et al. (1976), Lohman \& Kyllonen (1983) and Caplan \& Roman's (1998) compilations are some of these to name few. An expansive discussion on these tests is beyond the scope of this study. 


\section{How People Create Mental Models During Navigation}

Spatial ability of an individual comes to play when the user performs the task of navigation in information space. For instance, authors of an information space possess a semantic structure of information pieces that primarily resides in their mind. The virtual space that is created by unifying these models of one or more authors is not more than a transient representation of the whole information. A reader or information seeker, when comes to interact with the intermediate representation, tries to develop another mental model based on what is seen or found through the navigation. It is believed that if the information nodes are related spatially in some extent, both the authors and readers develop a space out of these nodes where they start navigating, unconsciously, during developing or traversing the virtual model (e.g. a WWW site). The shape of this space depends on the individual's various cognitive abilities where spatial ability is, presumably, the principal component (Benyon \& Murray, 1993).

Based on the model, a user tries to predict the hidden surfaces or parts of the space. Dillon (1991), in his article on readers' model on academic articles, showed results that support the existence of such a predictive model in each reader's mind during interaction with paper or digital journal articles. The accuracy of this prediction depends on how the reader design the structure based on experience and various cognitive abilities. Our principal focus is on finding the influence of user's spatial ability in creating such a model and how information can be presented to user so that the user can predict the original structure of information better and, essentially, enjoys effective navigation.

\section{Navigation and Spatial Organization of Information}

Background studies, such as those by Allen (1998), show that spatial organization of information visualization helps in learning and memory. As short term memory plays a key role in navigation sessions (Hewett, 1998), spatial organization of information, eventually, results in better navigation in information space. Again, long-term memory encapsulates experiences and habits in human brain (Tulving, 1983; Coone \& Fisher, 1998). Chen (2000) identified experience with the system as the main factor for lost less navigation. Taken as a whole, spatial organization of information has received much focus in finding the exact relation between spatial ability and conceptualization of information space. Stanney et al. (1995) found that visual mediators, such as $2 \mathrm{D}$ and $3 \mathrm{D}$ organization of information, remove the discrimination of mental models between people with low and people with high spatial ability. Similar results were found where Allen (1998) showed that it was not the high spatial people, but rather those with low spatial ability who got benefited from the spatial representation of information. Some of Allen's result was interesting where high spatial people's performance degraded due to the presence of spatial layout of information. Höök \& Dahlbäck (1997) also studied that visual momentum in information space helps with low spatial people. It is still not established firmly that how exactly high spatial people affected by spatial representation of information.

In the next section we discuss on the framework that is designed to find answers 
Figure 1 An example of regular breadcrumbs

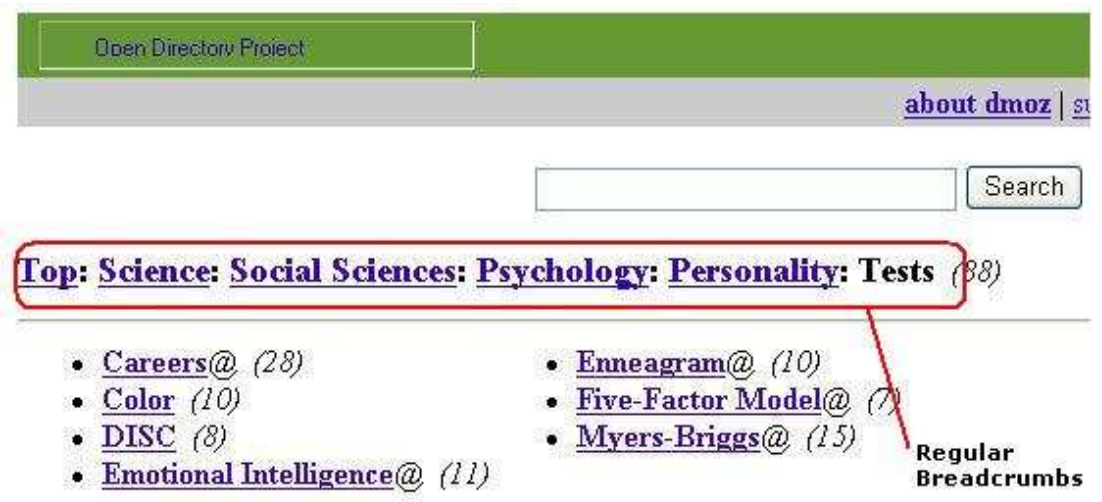

to these questions. We choose the World Wide Web as the information space for navigation because of its complexity and enormosity.

\section{Research Framework}

The purpose of this research is to gain a better understanding of how a special navigational tool called Breadcrumbs in the World Wide Web (WWW) sites helps people in their information seeking and keep them away from the state of being lost (Smith, 1986). Breadcrumbs, in general, are a list of hyperlinks that "convey information to the user (about the site structure or the path they have taken), and to also give users a way to select links from the breadcrumbs (in order to go "up' in the site hierarchy or to re-trace their steps)" (Instone, 2002). Breadcrumbs have evolved as a major navigational tool of the WWW, but there has been little research regarding their "precise concepts and terminology in order to effectively use breadcrumbs in different situations" (Instone, 2002).

Instone (2002) distinguished three types of breadcrumbs: location, path and attribute breadcrumbs. Location breadcrumbs show readers where the current web page is in the hierarchy of the current website. As such, they are rather like route markers on a highway, which are consistent with author's model. Path breadcrumbs show readers the sequence of web pages that they visited at the current site to 'arrive at' the current web page. Sometimes, a page can contain several paths of breadcrumbs about other pages that share some common attributes with the current page. These are attribute breadcrumbs. From the definition, it is clear that location breadcrumbs give user an idea of where exactly they reside in the site hierarchy. This helps in better understanding the structure of that particular information space. Throughout the rest of this framework, the term 'regular breadcrumbs' $(R B C)$ will generally mean location breadcrumbs if not specifically indicated.

Many examples of regular location breadcrumbs can be found in websites. Figure 1 shows an example of regular breadcrumbs.

Hochheiser et al. (1999) investigated the performance benefits of simultaneous over sequential menus and found that sequential menus are better suited for in- 
Figure 2 An example of experimental breadcrumbs

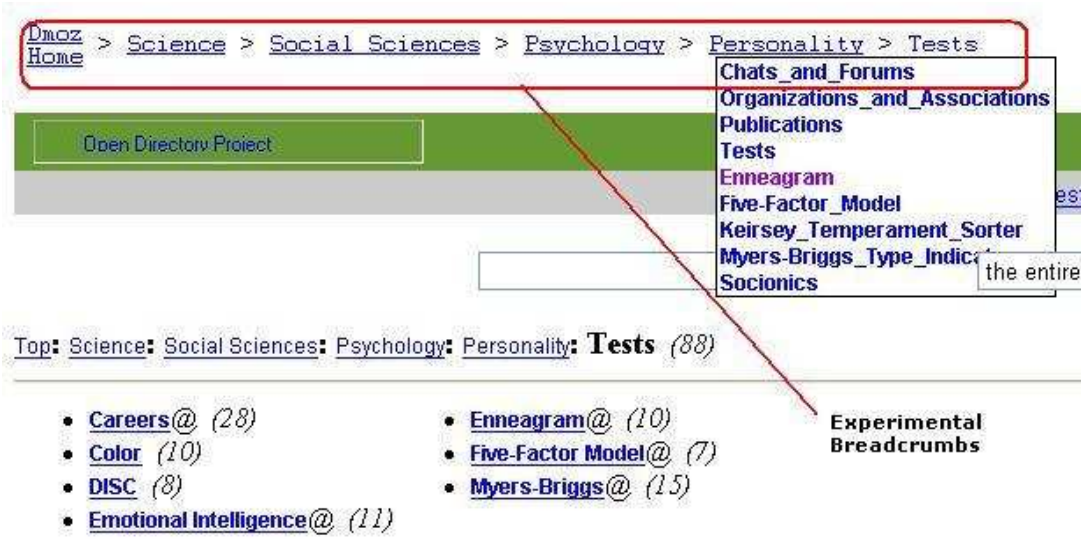

formation query where some kind of hierarchy exists (Hochheiser et al. 1999). In another study by Zaphiris et al. (1999), in-place expandable menus in web environment present a hierarchy of choice to users and reduce backtracking and lostness. The experimental breadcrumbs $(E B C)$, that we developed, provides both simultaneous and sequential features for a website which is an ideal combination for such navigation tasks. The experimental breadcrumbs look like regular breadcrumbs at the first place; but there is a menu associated with each of the breadcrumbs and it pops up when user moves the mouse over that specific breadcrumb item. The menu contains all the links that are one level deeper in site hierarchy of that particular item. Figure 2 shows an instance of experimental breadcrumbs.

Neerincx \& Lindenberg (1999) abridged on three types of spatial navigation support that help people in wayfinding in large information space. These are landmarks, history map and navigation assistant. Landmarks are the navigation cues that help in user in recognizing their situation in the search space. A history map keeps a trail of users navigation activities. A navigation assistant has the knowledge of the domain and is able to dynamically provide advice to the individual user by analyzing various individualistic factors like interest, browse history, profession, education and age.

In experimental breadcrumbs, the breadcrumb trail shows the history of the user's navigation activities. The pop-up menu is dynamic in the sense that it always directs users where else they can go from that specific breadcrumb item. The definition of experimental breadcrumbs, itself, contains the landmark feature of an ideal navigation tool. As the links in the menus are exactly one level down the site hierarchy, it gives users the awareness regarding their situation during navigation. Allen's (1998) work on spatial information presentation clearly shows the effectiveness of $2 \mathrm{D}$ spatial layout of information for low spatial ability people in their navigation in information space. The $2 \mathrm{D}$ representation style of the experimental breadcrumbs, hence, reduces the limitation of regular linear breadcrumbs that might be experienced by low spatial ability people. 


\section{Experiment}

To investigate the utility of the research framework, we designed an interactive experiment using human participants. Each participant signed a consent form stating that they were voluntarily participating in the experiment. They filled up a preliminary questionnaire, which included information such as age, sex, education and familiarity with the Web. Before performing navigation tasks, each participant took part in two paper-pencil based tests to assess spatial ability. The navigation part of the study used two conditions of task set. Each task was to reach a destination page, as the question demanded, in a large hierarchical website through navigation. Each condition had four such tasks with alternating sequence of presence of regular and experimental breadcrumbs in a cross-over design. The first two tasks were specified as practice session and the last two as the test session. The participants were assigned to either of the two conditions in predetermined sequence so that the population for each condition remained balanced. After completion of task sets, participant was requested to fill out a post-experimental questionnaire. The post-experimental questionnaire contained questions; asking participants to rate initial instruction, use of breadcrumbs, aspects of the website such as format, ease of use, ease of navigation, speed of download, and satisfaction on a five-point Likert scale.

\subsection{Website and Data Extraction}

The study required a large hierarchical website where a meaningful hierarchy exists. Open Directory Project site (http://www.dmoz.org) is such a site. It contains more than 4 million site references having around 64,739 editors and over 590,000 categories. These statistics were found from the downloaded copy of the site for the experiment. The whole site was downloaded using HTTrack, a website copier (http://www.httrack.com) and 'Mutator', a web crawler developed for this study. The web pages for this experiment are permitted for use according to the free use license of Open Directory Project. Once copied, we reproduced a different version of the whole Open Directory Project site in which experimental breadcrumbs were inserted in each page. Both the downloaded and modified sites were hosted at the Unix server 'Flame' of Faculty of Computer Science, Dalhousie University. Two sub-domains were created under Faculty of Computer Science website to access the sites. The site with regular breadcrumbs was hosted under the 'dmoz' sub-domain of faculty website (http://dmoz.cs.dal.ca). The other site with experimental breadcrumbs was accessed using 'mdmoz' sub-domain of the same parent website (http://mdmoz.cs.dal.ca).

\subsection{Participants}

The twenty-six unpaid volunteer participants of the experiment were graduate $(n=16)$ and under graduate $(n=10)$ students of Dalhousie University. Sixteen participants were from Computer Science discipline and seven were from Faculty of Science and the rest were from other disciplines. The spread of age range was from $\leq 20(n=1)$ to $41-50(n=1)$ with the age range $21-25$ years having the highest 
frequency (namely 12). There were nineteen male and seven female participants and all of them were familiar with the WWW environment and spend an average of 6-10 hours on the Internet each day. One participant faced a problem in understanding the language of the website and hence, the result from that participant was excluded from the final analysis. Recruitment of participants consisted of inclass recruitment with permission from instructors, in-person and email message to the students' group-mail.

\subsection{Spatial Ability Tests}

In this study, spatial visualization ability was assessed using the paper-pencil based paper folding test (VZ-2) and surface development test (VZ-3) taken from the Kit of Factor-Referenced Cognitive Tests (Ekstrom et al., 1976).

\subsection{Data Acquisition}

For data acquisition purpose, a customized usability testing service Uzilla ${ }^{a}$ was used in the study. Uzilla is an instrumented Web browser that logs user interaction details like clicks, mouse downs, mouse overs and scrolling with the internet based systems into its integrated, analysis and aggregation data collection server (Edmonds, 2003).

\subsection{Independent Variable}

Spatial ability was the independent variable that has been focused in this study. Spatial ability score for each participant was the sum of scores on the VZ-2 and VZ-3 tests. Answers were corrected for guessing following the equation described in the Manual for the Kit of Factor-Referenced Cognitive Tests (Ekstrom et al., 1976). Skewness and kurtosis of the distribution were calculated using statistical functions available in Microsoft Excel 2003. The skewness of the distribution was 0.18 . The value, being close to zero, meant that the distribution was not skewed and did not have any heavy left or right tail. The kurtosis score was -0.83 . According to the analysis, an ideal normal distribution should have a kurtosis score equal to zero. The negative value indicated that the distribution was not peaked but rather was relatively flat. There were no statistics available on these tests in Kit of Factor Referenced Cognitive Tests (Ekstrom et al., 1976). However, a previous compilation of this kit by French et al. (1963) contains statistics on these tests from two studies. The first study had 46 college students as participants. The mean score was 28.7. Another study, found in the same (French et al., 1963), used 86 Army enlistees as participants. The mean score in this study was 24.1. The mean score for this study was 21.67

Of the twenty-six participants, there were 13 participants for both Condition 1 and 2. The participants were partitioned into two groups: 'low' and 'high' by the median (21.38) of the spatial ability scores. Thirteen participants above the median were placed in the high spatial ability group and 13 participants below the

\footnotetext{
ahttp://www.uzilla.net
} 
median were placed in the low spatial ability group. However, after classification, for Condition 1, there were 7 participants in the low spatial ability group and 6 participants in the high spatial ability group. For Condition 2, there were 6 participants in the low spatial ability group and 6 participants in the high spatial ability group. For this condition, one participant who faced problem in understanding the language of the website, was not considered for the classification.

\subsection{Dependent Variables}

Speed: Speed was calculated from the time taken (in seconds) to complete a task. A low score in this measure indicated more speed.

Accuracy: The accuracy or effectiveness measure was quite straight forward. Accuracy value $(A)$ was 1 for each task participant completed successfully and 0 otherwise.

One accuracy measure was calculated for each group of participants for each task. The measure was the proportion of number of times participants completed the task accurately and total number of participants in the group.

Lostness: Lostness $(L)$ is a scale composed of the number of unique and total pages visited by the participant in comparison to the optimal number of page visit required to complete the task (Smith, 1996). In this study, the optimal number refers to the minimum number of page views required for the task. Smith described an user as definitely 'lost in a hyperspace' if the lostness score was greater than 0.5 and any score less than 0.4 as 'not lost' (Smith, 1996).

To calculate the score she proposed the following equation:

$$
L=\sqrt{(D / T-1)^{2}+(R / D-1)^{2}},
$$

Where

$T$ Total number of pages visited by a participant in a task.

$D$ Number of distinct pages visited by a participant in a task.

$R$ Minimum number of page visits required to complete a particular task.

Efficiency: Efficiency $(E)$ deals with the total number of page views versus the minimal or optimal number of page views and the accuracy of task completion. It is correlated with lostness but not perfectly, as users with the same lostness may vary in efficiency (Smith, 1996). Efficiency measure was calculated using another equation proposed by Smith. A low score in this scale indicated more efficiency. The equation is as follows:

$$
E=\sqrt{(D / T-1)^{2}+(R / D-1)^{2}+(A-1)^{2}} .
$$

Tool Selection Preference: Participants differed based on their preference of selecting navigation tool. For this measure, the percentage of clicks on the Back button $(B C)$ with respect to total clicks $(T C)$ were calculated. The goal of this analysis was to find out how did the users selected or moved away from the Back button based on the available tools for a particular task. 


\section{Results}

The analysis was carried out using the statistical analysis software, SAS ${ }^{\circledR}$ v-9.1. The standard procedure MIXED in SAS ${ }^{\circledR}$ was used to conduct the analysis. The procedure used two-way repeated measure analysis of variance to test the hypotheses. As the task settings were identical except the type of breadcrumbs present, Breadcrumb type was a another factor in the experiment. It took either of two values: $R B C$ or $E B C$ based on the breadcrumb type present in the task. The analysis used breadcrumb type as the within-subject factor and spatial ability as the betweensubject factor and $\alpha$ was set at 0.05 . The analysis also investigated the interaction effect between spatial ability factor and the other independent factor: breadcrumb type, in order to explain the results in more detail. It was assumed that the data values of each dependent measures, taken in the experiment, followed a normal distribution and there was no hidden pattern in the residuals of the values. Model diagnostics in $\mathrm{SAS}^{\circledR}$ were run to see if there were any underlying patterns in the data points. The analysis showed no noticeable departure from the assumptions.

\subsection{Speed}

The statistical analysis about the effect of spatial ability on speed did not reveal any significant result. Although it is found that participants with high spatial ability had less mean speed $(463.6 \mathrm{sec}$.) than that $(525.2 \mathrm{sec}$.) of participants with low spatial ability, the difference was not significant. Furthermore, there was no significant interaction effect between spatial ability and breadcrumb type.

\subsection{Lostness}

No significant effect of spatial ability on lostness was found from the analytic statistics. Although the statistics on lostness show that participants with high spatial ability were less lost on average (0.75) than participants with low spatial ability (0.81), the average difference was not statistically significant. In addition, there was no interaction effect found between spatial ability and breadcrumb type.

\subsection{Efficiency}

Participants with high spatial ability were more efficient (i.e. had low efficiency scores) than the participants with low spatial ability. The standard deviations in the efficiency scores of two groups of participants were close and the values were almost equally dispersed. Table 1 presents the descriptive statistics on both groups of participants.

After running the analytic statistical procedure, a significant main effect of spatial ability was found on the efficiency of the participants ( $d f=1, F$-value $=4.38, \operatorname{Pr}>F=0.0486)$. This value rejected the null hypothesis that spatial ability would have no effect on the efficiency of participants. A further analysis on interaction of spatial ability with condition resulted no significant outcome. The Least Square Means were calculated for two groups of participants and are presented in Table 2. Participants with high spatial ability had an estimated mean 


\begin{tabular}{l|c|c}
\multicolumn{2}{c}{ Spatial Ability Score } \\
& Low & High \\
\hline Mean & 1.05 & 0.84 \\
Standard Error & 0.07 & 0.08 \\
Median & 1.07 & 0.89 \\
Standard Deviation & 0.35 & 0.38 \\
Range & $0.17-1.51$ & $0.00-1.41$ \\
\hline \hline
\end{tabular}

Table 1 Descriptive statistics about the effect of spatial ability on efficiency

efficiency score 1.05. The estimated mean from the Least Square Means analysis for participants with low spatial ability was 0.85 .

\begin{tabular}{c|l|c|c|c|c} 
Spat. Ab. & $\begin{array}{l}\text { Est. Mean } \\
(\text { Score })\end{array}$ & DF & $t$ Value & $\operatorname{Pr}>|t|$ & Range (Score) \\
\hline Low & 1.05 & 21 & 15.46 & $<.0001$ & $0.91-1.19$ \\
\hline High & 0.85 & 21 & 11.99 & $<.0001$ & $0.70-0.99$ \\
\hline \hline
\end{tabular}

Table 2 Least Square Means statistics on efficiency about the main effect of spatial ability

\subsection{Tool Selection Preference}

Descriptive statistics about the effect of spatial ability on tool selection preference are shown in Table 3. Participants with high spatial ability used the Back button less than the participants with low spatial ability.

\begin{tabular}{l|c|c} 
& Low Sp. (\%) & High Sp. (\%) \\
\hline Mean & 20.3 & 12.9 \\
Standard Error & 3.0 & 2.0 \\
Median & 19.1 & 8.6 \\
Standard Deviation & 15.2 & 10.0 \\
Range & $0.0-41.8$ & $0.0-34.0$ \\
\hline \hline
\end{tabular}

Table 3 Descriptive statistics about the effect of spatial ability on percentage of Back button click

The difference in using Back button in Table 3 came as marginally significant after the hypothesis testing $(d f=1, F$-value $=4.16, \operatorname{Pr}>F=0.054 ; d f=21$, $t$-value $=2.12, \operatorname{Pr}>|t|=0.0462)$. Although $\operatorname{Pr}>F$ is marginally greater than the significance level (0.05), the $t$-value and $\operatorname{Pr}>|t|$ reported by the procedure MIXED rejected the null hypothesis that spatial ability would have no effect on tool selection preference. Least Square Means were calculated for both group of participants and are presented in Table 4 . There was no significant interaction effect found between spatial ability and breadcrumb type. 


\begin{tabular}{r|ccccl} 
Spatial Ability & Est. Mean (\%) & DF & $t$-value & $\operatorname{Pr}>|t|$ & Lower-Upper (\%) \\
\hline Low & 20.6 & 21 & 7.90 & $<.0001$ & $15.1-26.0$ \\
High & 12.9 & 21 & 4.78 & 0.0001 & $7.3-18.5$ \\
\hline \hline
\end{tabular}

Table 4 Least Square Means statistics on percentage of Back button click about the main effect of spatial ability

\subsection{Accuracy}

Accuracy score of a participant was 1 if the task was completed successfully, 0 otherwise. An overall accuracy score was calculated, using the accuracy scores for the tasks performed by low spatial ability participants, by taking proportion of total number of success in task completion and total instances of such tasks. Another similar score was calculated using the accuracy scores for the group of participants with high spatial ability. Table 5 shows the result of accuracy score between two groups of participants.

\begin{tabular}{cc|c} 
& & Overall Accuracy Score \\
\hline Tasks by Low Sp. & $(N=26)$ & 0.50 \\
Tasks by High Sp. & $(N=24)$ & 0.79 \\
\hline \hline
\end{tabular}

Table 5 Accuracy measure of participant groups with low and high spatial ability

Participants with high spatial ability were more successful than participants with low spatial ability in terms of the overall accuracy score of the group.

\section{Discussion}

The analysis in the previous section revealed that spatial ability had a significant main effect on the efficiency. Participants with high spatial ability were more efficient than participants with low spatial ability. Besides, participants with high spatial ability used the Back button less than participants with low spatial ability and difference came as marginally significant. The main effect was absent for the speed and lostness measures. However, this outcome supports the previous studies on other information spaces stating that spatial ability influences navigation performance in information space. Further analysis showed that, participants with high spatial ability had more overall accuracy score than participants with low spatial ability in all task arrangements. However, due to the nature of these measures, no statistical significance analysis was done and the results can be taken as informal but guiding towards future prediction. This study also investigated the relationship between the spatial layout of breadcrumbs and spatial ability of people. The spatial layout of experimental breadcrumbs did not affect the participants with high and low spatial ability which might have indicated that breadcrumbs can be accepted as a navigation tool for people with wide variety of spatial ability. 


\section{Conclusion}

In this study, we have reviewed previous efforts in explaining the relation between spatial ability and navigation in information space. We proposed a tool for navigation in large hierarchical websites. We found significant difference between high and low spatial ability participants in navigation efficiency. The result also indicated that low spatial ability participants preferred browser's Back button more than high spatial ability participants. This result will help in the development of future Web browsers. However, the experimental tool did not interact with spatial ability in navigation performance which indicated that more spatial concepts like spatial hypertext can be investigated in web based systems with intense attention. Finally, it seems clear from this study that additional efforts are needed to come up with more effective and usable navigation scheme for people with diverse individual differences.

\section{References and Notes}

1 Allen, B., 1998, 'Information space representation in interactive systems: Relationship to spatial abilities', Proceedings of the third ACM conference on Digital Libraries, Pittsburgh, PA, USA, pp. 1-10.

2 Benyon, D., 2001, 'The New HCI? Navigation of Information Space', KnowledgeBased Systems 14(8), 425-430.

3 Benyon, D. and Murray, D., 1993, 'Developing Adaptive Systems to Fit Individual Aptitudes', Proceedings of IWIUI, Orlando, FL, USA.

4 Caplan, B. and Romans, S., 1998, Neuropsychology, Plenum Press, New York, USA, chapter 'Assessment of Spatial Abilities', pp. 379-419.

5 Chen, C., 2000, 'Individual differences in a spatial-semantic virtual environment', Journal of the American Society for Information Science 51(6), 529-542.

6 Coone, L. and Fisher, R., 1998, 'Cognitive psychology on memory', Technical Report, (Available at: http://www.scism.sbu.ac.uk/inmandw/tutorials/memory/g1.htm).

7 Dahlbäck, N., Höök, K. and Sjölinder, M., 1996, 'Spatial Cognition in the Mind and in the World: the Case of Hypermedia Navigation', In Proceedings of CogSci96, July, San Diego, CA, USA.

8 Dahlbäck, N. and Lönqvist, P., 2000, 'Navigation and Learning - A Cognitive Analysis of User Tasks in Electronic Information Spaces', Short paper in CHI '2000, Amsterdam, Netherlands.

9 Dieberger, A., 1997, 'Navigation metaphors and social navigation in information spaces', Position paper for the Conference on Computer Human Interaction (CHI)'97.

10 Dillon, A., 1991, 'Readers models of text structures: the case of academic articles', Man-Machine Studies 35, 913-925.

11 Dillon, A., 1995, 'What is the Shape of Information? Human factors in the development and use of digital libraries', SIGOIS Bulletin 16(2), 32-34.

12 Dillon, A. and Vaughan, M., 1997, 'It's the journey and the destination: Shape and the emergent property of genre in evaluating digital documents', New Review of Multimedia and Hypermedia 3, 91-106. 
13 Egan, D., 1988, Handbook of Human Computer Interaction, chapter 'Individual Differences in Human-Computer Interaction', Amsterdam, The Netherlands.

14 Ekstrom, R., French, J., Harman, H. and Dermen, D., 1976, Manual for kit of factor referenced cognitive tests, Educational Testing Service, Princeton, NJ, USA.

15 French, J., Ekstrom, J. and Price, L., 1963, Kit of Reference Tests for Cognitive Factors, Educational Testing Service, Princeton, NJ, USA.

16 Hewett, T., 1998, 'Cognitive factors in design: Basic phenomena in human memory and problem solving', Proceedings of the Conference on Computer Human Interaction (CHI), pp. 117-118.

17 Hochheiser, H., Kositsyna, N., Ville, G. and Shneiderman, B., 1999, 'Performance benefits of simultaneous over sequential menus as task complexity increases', Technical Report CS-TR-4066, (Available at: http://citeseer.ist.psu.edu/article/hochheiser00performance.html)

18 Höök, K. and Dahlbäck, N., 1997, 'Designing navigational aids for individuals', Submission to CHI97 Workshop on Navigation in Electronic Worlds, March 23-24, Atalanta, GA, USA.

19 Instone, K., 2002, 'Location, path and attribute breadcrumbs', Proceedings of the 3rd Annual Information Architecture Summit, March 16-17, (Available at: http://keith.instone.org/breadcrumbs)

20 Lohman, D. and Kyllonen, P., 1983, Individual Differences in Cognition, Vol. 1, Academic Press, chapter 'Individual Differences in Solution Strategy on Spatial Tasks', pp. 105-135.

21 Neerincx, M. and Lindenberg, J., 1999, 'Supporting individual situation awareness in web-environments', Proceedings "Ergonomie in uitvoering" Conference, http: //www.cwi.nl/projects/uwish/papers/sapaper.pdf, The Netherlands, November $12-13$.

22 Robertson, G., 1997, 'Navigation in information spaces', Submission to CHI97 Workshop on Navigation in Electronic Worlds, research.microsoft.com/ ggr/ navigation/navigation.doc, March 23-24, Atalanta, GA, USA.

23 Sjölinder, M., 1996, 'Individual Differences in Spatial Cognition and Hypermedia Navigationf', Towards a Framework for Design and Evaluation of Navigation in Electronic Spaces, Swedish Institute of Computer Science, pp. 61-72.

24 Smith, P. A., 1996, 'Towards a practical measure of hypertext usability', Interacting with Computers 8(4), 365-381.

25 Stanney, K. and Salvendy, G., 1995, 'Information visualization: Assisting low spatial individuals with information access tasks through the use of visual mediators', Ergonomics 38(6), 1184-1198.

26 Tulving, E., 1983, Elements of episodic memory, Oxford [Oxfordshire], New York: Clarendon Press; Oxford University Press.

27 Edmonds, A., 2003, 'Uzilla: A new tool for Web usability testing', Behavior Research Methods, Instruments, \& Computers, 35(2), pp. 194-201.

28 van Dyke Parunak, H., 1989, 'Hypermedia Topologies and User Navigation', Proceedings of the Conference on Hypertext, pp. 43-50.

29 Vicente, K. and Williges, R., 1988, 'Accomodating individual differences in searching a hierarchical file system', International Journal of Man Machine Studies 29, 647688.

30 Zaphiris, P., Shneiderman, B. and Norman, K., 2002, 'Expandable indexes versus sequential menus for searching hierarchies on the world wide web', International Journal of Behaviour and Information Technology 21(3), 201-207. 